spouses and family of depressed patients also suffer terribly, and must be partners in the treatment. His cogent discussion of the classification of mood disorders and the current diagnostic process and measurements of disease, by categorical and dimensional measures, respec-

tively, is a godsend. I found his discussion of the nature and seriousness of postnatal depression particularly valuable. He also does a very good job of summarizing the neurobiology of depression, with an emphasis on the role of alterations in serotonergic and noradrenergic neuronal systems, and of the hypothalamic-pituitary-adrenal axis in the pathophysiology of mood disorders.

What didn't I like? The author's understanding of the data from controlled clinical trials related to the treatment of depression was a bit disappointing. Perhaps I expected too much, but we get a relatively non-critical and superficial view of a very complex field. For example, Wolpert says that the literature reveals all antidepressants to be equally efficacious. Although this is technically true, in that no regulatory agency in any country has ever recognized one antidepressant as more effective than another, there is a raging controversy in the field over this issue. There are data that suggest that certain of the older tricyclic antidepressants, such as chlorimipramine, are more effective than some of the newer agents. This is obviously not a trivial point, and may relate to the older drugs' effects on more than one neurotransmitter system, compared with the newer, more selective agents.

Perhaps more important is the lack of communication of the following observation, which is well known among psychiatrists: most depressed patients treated with an antidepressant respond only partially or not at all. Unfortunately, many general practitioners and even psychiatrists do not use measures of depression severity to follow their patients' progress, and therefore never realize that their patients have, in fact, not completely recovered. It is also important to determine whether the depressed patient has delusional (psychotic) thinking; such patients do not respond to antidepressant monotherapy, and require combination treatment with an antidepressant and an antipsychotic medication.

Another minor disappointment is Wolpert's discussion of the role of psychotherapy in treating depression, and the (perhaps) related issue of placebo response of depressed patients. It is difficult for the uninitiated to understand how depression can, on the one hand, be as severe as it is described (severe functional impairment, increased risk of heart disease and stroke, and increased risk of suicide) and, on the other, respond relatively well to placebo treatment. The answer lies, at least in part, in that the depressed patient assigned to the placebo group receives all of the treatment except the drug (the contact with a myriad of research personnel will be a form of therapy). In terms of the psychotherapies themselves, suffice it to say that the more severe the depression, the less likely that it will respond to psychotherapy alone. Moreover, as Wolpert points out, the decreases in concentration and cognitive dysfunction characteristic of severe depression hardly make the patient a candidate for psychotherapy. He aptly points out the important theoretical underpinnings of psychoanalysis and psychoanalytically oriented psychotherapy in the aetiology and treatment of depression, but surprisingly fails to highlight the lack of evidence for the efficacy of this approach.

I was not surprised by his attraction to genetics, as this field will probably shed considerable light on depression research over the next decade, perhaps more than any other field. The completion of the Human Genome Project will provide the field with tools that will surely advance depression research. I believe that functional brain imaging will also contribute much, as will the burgeoning pipeline of promising therapies.

In summary, Malignant Sadness will stand up well with other personal descriptions of depression, including some he refers to in his volume: Kay Redfield-Jameson's An Unquiet Mind and Kathy Cronkite's On The Edge of Darkness. It is a fitting companion to others who have written eloquently on this subject, including Tracey Thompson's The Beast: A Reckoning with Depression and Martha Manning's Undercurrents. In view of the growing evidence for the role of adverse early experience in the pathogenesis of depression, the only solution may be to offer Wolpert a sabbatical to share his developmental biology expertise, together with his personal knowledge of this disorder, to help design experiments to uncover biological causes of this observation.

Charles B. Nemeroff is in the Department of

Psychiatry and Behavioral Sciences,

Emory University School of Medicine,

Atlanta, Georgia 30322, USA.

\title{
Starr book wins Los Angeles Times prize
}

Douglas Starr's book Blood: An Epic History of Medicine and Commerce was announced as the winner of the science and technology category of the 1998 Los Angeles Times Book Prize last Friday (23 April). Starr was one of five finalists in the science and technology category:

\section{The Baltimore Case: A Trial of Politics, Science and Character}

by Daniel J. Kevles

W. W. Norton, $\$ 29.95, £ 21$

"Daniel Kevles performs a signal service by applying his historian's acumen to the mountain of documents accumulated by all those investigations, and to his own detailed interviews with the participants, to give us an orderly narrative of the whole affair. He did so, he says, because it seemed to him likely to 'throw some light on science in late-twentieth-century American society." Jon Turney, Nature 395, 30-31 (1998).

\section{Phantoms in the Brain: Probing the Mysteries of the Human Mind} by V.S. Ramachandran and Sandra Blakeslee William Morrow, \$27, £17.99

"Phantoms in the Brain is structured around the life histories of patients with unusual, often bizarre, neurological disorders. The narrative structure of biography has undoubted pedagogic value, but the worst examples of the genre come uncomfortably close to modern-day equivalents of a travelling freak-show. To their credit, this pitfall is skilfully avoided by Ramachandran and Blakeslee, who keep the ideas they are seeking to communicate centre stage." Raymond J. Dolan, Nature 396, 639-640 (1998).

\section{Taking Wing: Archaeopteryx and the Evolution of Bird Flight}

by Pat Shipman

Simon \& Schuster, \$25.

"Shipman makes an outstanding analysis of the contribution of Archaeopteryx to discussions about the origin of flight." Jose Luis Sanz, Bernardino P. Perez-Moreno \& Francisco J. Poyato-Ariza, Nature 393, 32-33 (1998).

\section{Blood: An Epic History of Medicine} and Commerce

by Douglas Starr

Knopf, \$27.50, £20

"While the value of a barrel of crude oil has fallen to $\$ 12$, an equivalent amount of blood is now worth around $\$ 60,000$. How this came about is lucidly recounted by Douglas Starr in this history of blood transfusion. The book is accessible to a wide-ranging audience." Fred S. Rosen, Nature 398, 303-304 (1999).

\section{Mendel's Dwarf}

by Simon Mawer

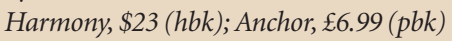

